\title{
Large eddy simulation of Cambridge bluff-body coal (CCB2) flames with a flamelet progress variable model
}

\author{
Jiangkuan Xing ${ }^{\mathrm{a}}$, Kun Luo ${ }^{\mathrm{a}, *}$, Yiran Chen ${ }^{\mathrm{b}}$, Oliver Stein ${ }^{\mathrm{c}}$, Andreas Kronenburg ${ }^{\mathrm{c}}$, \\ Kai Hong Luo ${ }^{\mathrm{d}}$, Christian Hasse ${ }^{\mathrm{e}}$, Jianren Fan ${ }^{\mathrm{a}}$ \\ ${ }^{a}$ State Key Laboratory of Clean Energy Utilization, Zhejiang University, Hangzhou, 310027, China \\ ${ }^{b}$ Center for Combustion Energy, Tsinghua University, Beijing 100084, China \\ ${ }^{c}$ Institut für Technische Verbrennung, Universität Stuttgart, Herdweg 51, 70174 Stuttgart, \\ Germany \\ ${ }^{d}$ Department of Mechanical Engineering, University College London, Torrington Place, London \\ WC1E $7 J E, U K$ \\ ${ }^{e}$ FG Simulation of Reactive Thermo-Fluid-Systems, TU Darmstadt, Otto-Berndt-Str. 2, 64287 \\ Darmstadt, Germany
}

\begin{abstract}
In the present study, we report the first LES study of the Cambridge CCB2 coal flames, one of the target flames in CBC workshop, with an extended two-mixturefraction FPV model. The extended FPV model is based on two mixture fractions considering the volatiles and char off-gases. The normalized total enthalpy is used for the interphase heat transfer modelling. Turbulence-chemistry interaction (TCI) is treated with an assumed PDF approach. The results show that the present LES can generally capture the flow field and particle distribution, while there are considerable deviations in the $\mathrm{OH}$ prediction due to the boundary treatment of using a mixture of volatiles and carrier gas to replace the methane-containing mixtures in the primary and pilot flow, which indicates for such gas-assisted coal flames, the pilot fuel stream needs to be rigorously considered in the flamelet tabulation that be resolved by
\end{abstract}

\footnotetext{
${ }^{*}$ Corresponding author:

Email address: zjulk@zju.edu.cn (Kun Luo)
} 
extending the two-mixture-fraction model into a three-mixture-fraction model which will be studied in the future. The instantaneous Lagrangian particles histories show that the increasing of coal load has a negligible effect on devolatilization, but delays the char conversion.

Keywords:

Large eddy simulation, Coal combustion, FPV model, Cambridge coal flames

\section{Introduction}

Coal is expected to still play an important role in the energy structure in the foreseeable future due to its abundance and low cost [1]. Coal is mainly utilized via pulverized coal combustion technology (PCC) in the heat and power plant with significant levels of pollutant formation. Making a detailed knowledge of the physical and chemical mechanisms in PCC is of great significance for developing more efficient and clearer coal combustion technologies.

Computational fluid dynamics (CFD) has become an important tool for PCC since details of the chemistry and flow field can be obtained, which is usually unavailable experimentally. CFD of turbulent flows can be divided into the Reynoldsaveraged Navier-Stokes (RANS) simulation, large eddy simulation (LES) and direct numerical simulation (DNS). Among them, LES is thought to be a promising highfidelity approach to investigate PCC due to its advantages in computational efficiency and accuracy compared with DNS and RANS respectively[2]. PCC involves complex multi-physics and chemistry [3], such as devolatilization, char oxidation and turbulence-chemistry interactions (TCI), which makes high-fidelity simulation of PCC very challenging. Therefore, very recently, the combustion community has organized the Workshop on Measurement and Simulation of Coal and Biomass Con- 
version (CBC) [4] to promote the development of high-fidelity coal combustion modelling approaches. The workshop has identified a series of target flames, including the CRIEPI [5] and Cambridge [6,7] coal flames, which are used to evaluate coal combustion sub-models and sub-grid TCI models. The used devolatilization models have progressed from the simple single-step model $[8,9]$ to the directly-coupled Chemical Percolation Devolatilization (CPD) model [10-12]. The subgrid TCI models have progressed from simple eddy-diffusion-based models, i.e. eddy break up model [9], to more efficient models, such as flamelet (steady laminar flamelet (LSM) model and flamelet/progress variable (FPV) model) $[8,10,13]$ and probability density function (PDF) [14-16]. Although good agreement with the experimental data can be achieved, there are still considerable deviations in the gas species predictions. The Cambridge coal flames, including the CCB1 (without bluff-body) [6] and CCB2 (with bluff-body) [7] flames, have complex configurations with a swirled pilot flow, resulting in more complex turbulent flow and combustion compared with those of the piloted coal jet flames studied earlier [5], which brings a big challenge for the TCI modelling. There is only one LES study reported for CCB1, in which only nonreacting flow results are compared with the experimental data and then the effect of $\mathrm{O}_{2}$ concentration on the NO emission is explored [17]. To our best knowledge, there is no LES study reported for the benchmark CCB2 flames.

The FPV model for coal combustion is firstly proposed by Watanabe and Yamamoto [13]. In our previous work [18], an extended flamelet progress variable (FPV) model was developed by normalizing the mixture fraction ratios and the total enthalpy to reduce the interpolation error and modify the heat transfer model, and the model performance was assessed by comparing with the detailed chemistry predictions using a priori and a posteriori analyses for PCC in a laminar counterflow. However, the model performance has not been further validated against 
experimental data from complex turbulent PCC. The numerical investigation of the experimentally well-studied CCB flames is a logical and necessary step to enhance the knowledge on PCC-FPV-LES modelling. Therefore, the objective of the present study is to evaluate the extended FPV model on the benchmark CCB2 flames using LES, which is different from previous studies with the LSM [10] and traditional FPV model [8] and also the first LES/FPV report of this burner. The LES results are compared with the available experimental data, including the velocity profiles, $\mathrm{OH}$ and Mie scatter images. In addition, instantaneous Lagrangian particles are analyzed to study the particle histories in terms of devolatilization and char conversion.

\section{Numerical approaches}

\subsection{Coal particle modelling}

In the present study, coal particles are traced in a Lagrangian manner, where the particle mass, momentum, and energy equations are solved considering the interactions with the gas phase using two-way coupling terms. The coal particles are assumed to be composed of volatile, char and ash, neglecting the water content. The devolatilization and char oxidation processes are considered. The devolatilization process is calculated with the single-step kinetic model proposed by Badzioch and Hawksley [19], with kinetic parameters fitted from the CPD model predictions [20]. The devolatilization products are assumed to be composed of $\mathrm{CO}, \mathrm{CH}_{4}, \mathrm{C}_{6} \mathrm{H}_{6}, \mathrm{C}_{2} \mathrm{H}_{4}$, $\mathrm{H}_{2}$, and $\mathrm{N}_{2}$ as ref. [21], and their mass fractions are determined from the CPD pre-

dictions, elemental and energy conservation equations. The char oxidation rate is described with the classical Baum and Street model [22]. 


\subsection{Gas phase modelling}

The Favre-filtered conservation equations for mass, momentum, total enthalpy, mixture fractions, progress variable and variance of mixture fraction are solved for the gas phase in the Euler framework. The Smagorinsky model [23] is used to close the subgrid eddy viscosity. Due to the low turbulence of the primary flow and fine mesh resolution in the concerned regions, more than 0.92 of the turbulent kinetic energy can be resolved, therefore the effect of sub-grid turbulence model is quite limited here. Two-way coupling source terms are used to describe the gas and particle interactions [3]. The radiation effect is considered with the P1 model [25]. All governing equations are not introduced here for brevity, and interested readers can refer to the previous studies [8, 24].

The FPV model is based on two mixture fractions [18], $Z_{v o l}$ and $Z_{c h a r}$, for volatiles and char-off gases, respectively, which are defined as $Z_{v o l}=\xi_{\text {vol }} /\left(\xi_{\text {vol }}+\xi_{\text {pro }}+\xi_{\text {ox }}\right)$ and $Z_{\text {char }}=\xi_{\text {pro }} /\left(\xi_{\text {vol }}+\xi_{\text {pro }}+\xi_{\text {ox }}\right)$, where $\xi_{\text {vol }}, \xi_{\text {pro }}$ and $\xi_{\text {ox }}$ are the mass of the gas released from the devolatilization, char-off gases and the oxidizer stream. The volatiles and char-off gases have different elemental compositions, which have been considered during flamelet table generation (fuel boundary of the flamelet calculation which will be introduced later). There are some improvements compared with our previous LES/FPV study [18]. Firstly, to avoid the numerical interpolation issues, we transfer the two mixture fractions into their sum and the ratio of $Z_{\text {char }}$ to their sum, $Z=Z_{\text {vol }}+Z_{\text {char }}, X=Z_{\text {char }} /\left(Z_{\text {vol }}+Z_{\text {char }}+\epsilon\right)$, where $\epsilon$ is a small positive number used to avoid dividing by zero. Secondly, the normalized total enthalpy, $H e_{n o r m}$, is used to improve the interphase heat transfer modelling [24]. The progress variable, $Y_{P V}$, is defined as $Y_{\mathrm{PV}}=Y_{\mathrm{CO}_{2}}+Y_{\mathrm{H}_{2} \mathrm{O}}+Y_{\mathrm{H}_{2}}$ [18]. Then the thermo-chemical quantities can be parameterized as $\psi=\phi\left(X, Z, H e_{n o r m}, Y_{P V}\right)$. Under the framework of LES, the TCI 
are considered by integrating the thermo-chemical quantities in the flamelet table using a joint PDF approach with the assumption that $X, Z, H e_{n o r m}$, and $Y_{P V}$ are statistically independent. The presumed $\delta$-PDF is used to describe the distributions of $X, H e_{n o r m}$, and $Y_{P V}$, and the sub-grid distribution of the total mixture fraction $Z$ is modelled with a $\beta$-PDF. The $\beta$-PDF requires the sub-grid variance of mixture fraction to access the flamelet table, thus the Faver-averaged governing equation for $\widetilde{Z^{\prime \prime 2}}$ is solved as our previous study [29]. Finally, the Favre-filtered thermo-chemical quantities in the flamelet table are parameterized as $\widetilde{\psi}=\phi\left(X, \tilde{Z}, \widetilde{Z^{\prime \prime}}, \widetilde{H e_{\text {norm }}}, \widetilde{\left.Y_{P V}\right)}\right.$. Based on the estimated parameters, the current turbulent flame is within the thin reaction zones, not in the broken reaction zones. Therefore, the basic assumption of the flamelet model is reasonable here.

In the present work, the flamelet table is generated using the FlameMaster code [26] with the skeletal CRECK52 mechanism [27], which includes both light hydrocarbons and - different from GRI3.0 - also a representative tar species. The fuel boundary of the flamelet is set as $Y_{\text {fuel }, k}=\left(\xi_{v o l} Y_{v o l, k}+\xi_{\text {pro }} Y_{\text {pro }, k}\right) /\left(\xi_{\text {vol }}+\xi_{\text {pro }}\right)=$ $(1-X) Y_{v o l, k}+X Y_{p r o, k}$, where $Y_{v o l, k}$ is the mass fraction of each volatile component $k . \quad Y_{\text {pro }}$ is the mass fraction of the char-off gas (i.e. $\mathrm{CO}$ and $\mathrm{N}_{2}$ ) [13] , and can be calculated with $Y_{p r o, C O}=M_{C O} /\left(M_{C O}+0.5 \gamma M_{N_{2}}\right)$ and $Y_{p r o, N_{2}}=$ $0.5 \gamma M_{N_{2}} /\left(M_{C O}+0.5 \gamma M_{N_{2}}\right)$, where $M_{C O}$ and $M_{N_{2}}$ are the molecular weights of $\mathrm{CO}$ and $\mathrm{N}_{2}$, respectively, and $\gamma$ is the volume ratio of the $\mathrm{N}_{2}$ and $\mathrm{O}_{2}$ in the carrier gas $(7 / 3)$. The final five dimensional flamelet table has a gird resolution of $10 \times 26 \times 10 \times 26 \times 31$ for $X \times \tilde{Z} \times \widetilde{Z^{\prime \prime}} \times \widetilde{\text { enorm }_{\text {nor }}} \times \widetilde{Y_{P V}}$, respectively. To assess the effect of table resolution, a finer table with resolutions of $10 \times 50 \times 20 \times 50 \times 40$ is also tested, and the simulation results show little difference(see the supplementary materials), therefore, the coarse table is used for the following cases. 


\section{Experimental and numerical setup}

The CCB2 burner [7] features three inlet streams, the central jet flow carrying coal particles with a bluff-body, the pilot methane-air flow with an axial swirler and the air coflow. The coflow, primary and pilot flows have volume flow rates

of 14.4, 6.0 and $4.0 \mathrm{~m}^{3} / \mathrm{h}$, respectively. Velocity profiles were obtained by means of Laser Doppler Velocimetry, and two-dimensional OH PLIF images were also obtained through Laser-induced incandescence. Four conditions, including the non-reacting case (NRF7), pure pilot gas flame case (P1), and two coal flame cases (A1 and A3) with different coal loads, were measured. The coal loads in flame A1 and A3 are 0.15 and $0.46 \mathrm{~g} / \mathrm{s}$, respectively.

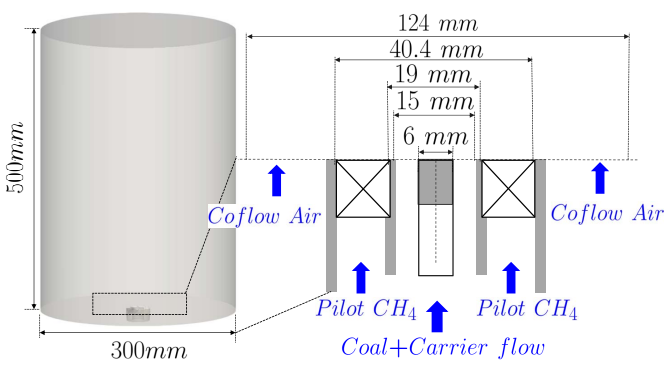

Figure 1: Schematic diagram of the computational domain and setup of the coal burner.

The computational domain and setup of the coal burner are shown in Fig. 1. The computational domain is a cylinder combustion chamber with a length of 500 $\mathrm{mm}$ and a radius of $150 \mathrm{~mm}$, which includes the swirl burner, with the geometry of the latter provided by the Cambridge group on their website [28]. The upstream annular flow between the bluff-body and the outer wall of the swirler is not a part of the combustion simulation. Instead, a fully-developed turbulent ring pipe flow is used as the velocity boundary for the primary flow. The mean velocity boundary without fluctuation is used for the pilot and coflow flow. The total cell number 
is 5.6 million with a resolution of $60 \mu \mathrm{m}-280 \mu \mathrm{m}$ for the primary flow region, and a uniform resolution of $400 \mu \mathrm{m}$ for the pilot flow region, and 240 cells along the azimuthal direction, which has been demonstrated to be sufficiently fine by a grid independency analysis. Since the two-mixture-fraction FPV model only consider the volatile and char-off gases, the methane in the primary and pilot flows needs to be assumed to be a mixture of volatiles and carrier gas for model implementation. We have determined the boundary conditions of the $Z_{v o l}$ for the primary and pilot flow to stay consistent with the energy input and volume flow rate in the experiment as our previous study [8]. The determined $Z_{v o l}$ for the pilot and primary flow boundary are 0.07 and 0.052 , respectively, and the total enthalpies for the pilot and primary flow are calculated based on $Z_{v o l}$. The particle sizes are assumed to be represented by a Rosin-Rammler distribution with a specific diameter of $40 \mu \mathrm{m}$ and a spread parameter of 2 as Ref. [17]. The fitted $A, E$ and $V^{*}$ of the single-step devolatilization model are $4.4269 \times 10^{4} \mathrm{~s}^{-1}, 4.101 \times 10^{4} \mathrm{~J} / \mathrm{kg}$ and 0.552 , respectively [30]. The mass fractions of volatile components are $0.312,0.142,0.439,0.0632,0.0185,0.0253$ for $\mathrm{CO}, \mathrm{CH}_{4}, \mathrm{C}_{6} \mathrm{H}_{6}, \mathrm{C}_{2} \mathrm{H}_{4}, \mathrm{H}_{2}$, and $\mathrm{N}_{2}$, respectively. To allow for the ignition of the pilot and primary flow, the domain outside the burner is initialized with a small but nonzero progress variable. It is worth noting that since the $\mathrm{H}_{2}$ fraction in the volatiles strongly affects the burning rate of the fuel mixture, the pilot gas flame resulted in flashback into the swirler in our early tests, resulting in strong over-predictions on the mean velocity profiles at the swirler outlet. To avoid this non-physical phenomenon, we artificially limit the rate of chemical reactions inside the swirler to zero.

Four cases, including the non-reacting, pure pilot gas flame and two coal flames with different coal loads, are simulated. All simulations are conducted with an inhouse solver developed based on the open-source CFD code OpenFOAM, which has been extensively validated with the experimental data of the CRIEPI coal jet flame 
in our previous studies $[8,18]$. The gas phase governing equations are solved with a finite volume method using the PIMPLE algorithm, and the time integration terms are discretized with a second-order implicit scheme. The Courant number is set to be less than 0.3 , resulting in a time step of $4 \times 10^{-6} \mathrm{~S}$ based on the pre-analysis of different time scales and pre-tests of different Courant numbers.

\section{Results and discussion}

\subsection{Non-reacting case}

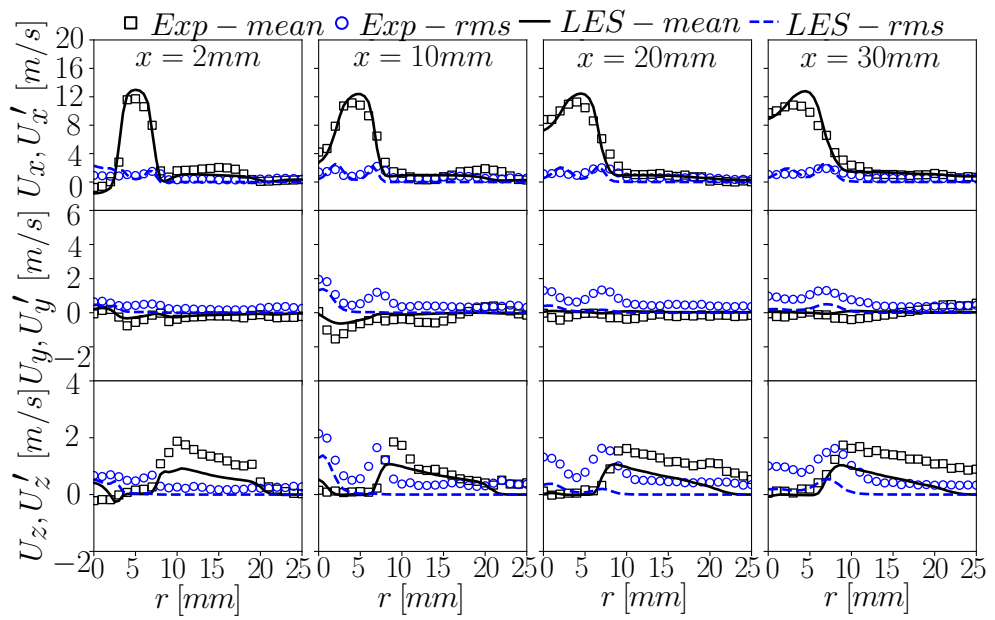

Figure 2: Comparisons of the mean and RMS velocities profiles of the LES results and experiments for the non-reacting case.

For the velocity measurement in the experiment [7], aluminum oxide $(1 \mu m)$ is used for the pilot and co-flow, and coal particles are used for the primary stream. At those near-nozzle measurement locations, there are very few particles transported to the outer radial regions $(r>10 \mathrm{~mm})$, which can be found in both the present LES results and the experimental Mie scatter images. Therefore, the "coal particle" velocity measured in the pilot and co-flow regions is actually the gas velocity. In 
addition, as coal particles are initially injected with the same velocities as the local fully-developed turbulent ring pipe flow, we assume the coal particle velocities are quite similar to those of the gas phase due to very limited particle residence time at the near-nozzle regions, which is also supported by the mean particle Stokes number (about 1.5). Therefore, for the consistency and continuity of the velocity statistics, the gas phase velocity profiles are used for the following comparisons. Figure 2 compares the experimental and LES results of the mean and RMS velocity profiles at downstream locations 2, 10, 20 and $30 \mathrm{~mm}$. The mean and RMS axial velocity profiles of LES results match well with the experimental results for these four downstream locations. While the RMS velocities in $y$ and $z$ directions are under-predicted, which may be attributed to the inlet boundary treatment of using a fully-developed turbulent ring pipe flow to characterize the bluff-body effect as well as the experimental uncertainties. At $x=2 \mathrm{~mm}$, the mean axial velocity becomes negative at the $r=0$, which indicates that there is a recirculation zone induced by the blocking of the bluff body. It is interesting to find that in the experiment, there is small velocity fluctuation at the pilot flow regions $(10 \mathrm{~mm}<r<20 \mathrm{~mm})$ although the pilot flow has a Reynolds number of 1520 (laminar flow), which can not be reproduced even a turbulent pipe ring inlet is used for the pilot flow.

\subsection{Reacting cases}

\subsubsection{Velocity comparisons}

Figure 3 shows the comparisons of the experimental and LES results of the mean and RMS velocities at different downstream locations for the pure pilot gas flame. Overall, good agreements on the mean and RMS axial velocities can be predicted. While an under-prediction of $U_{z}$ can be found at outer radial regions $(r>20 \mathrm{~mm})$, which indicates a under-prediction of the pilot flow swirling. It is also found that 


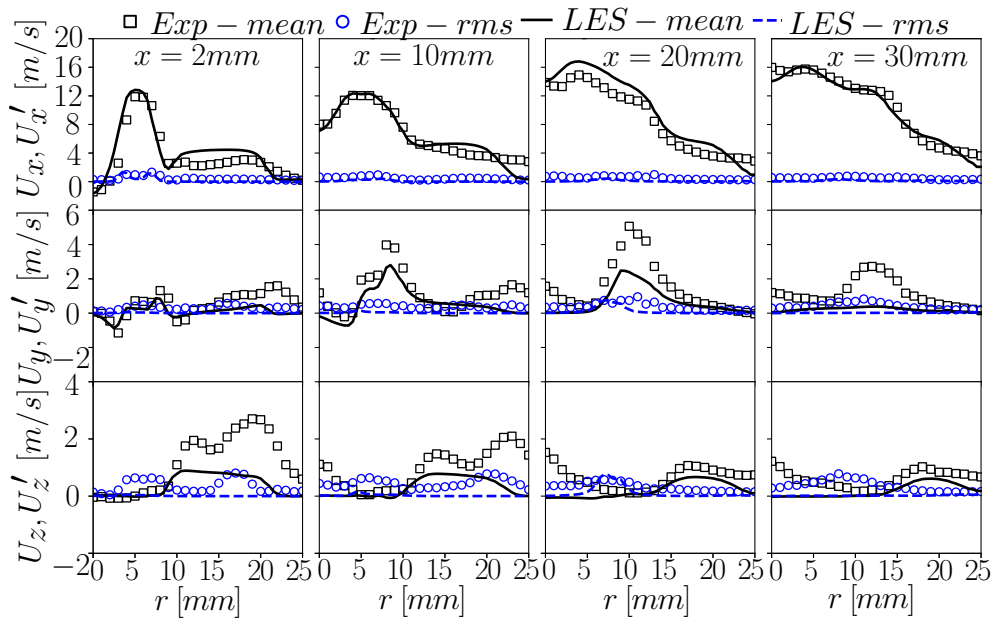

Figure 3: Comparisons of the mean and RMS velocities profiles of the LES results and experiments for the pure pilot gas flame case.

$U_{y}$ matches well with the experimental data at $x=2$ and $10 \mathrm{~mm}$, while is obviously under-predicted at $\mathrm{x}=20$ and $30 \mathrm{~mm}$. This indicates a compact flame located near the centreline, which can be attributed to the boundary treatment of assuming pilot gas as a mixture of volatiles and carrier gas, resulting in an under-prediction of flame angle and length, especially when heavy hydrocarbon is introduced in the volatile components $[31,32]$. RMS velocities at the outer radial region $(10 \mathrm{~mm}<r<20 \mathrm{~mm})$ are under-predicted, which may result from the under-prediction of the angle of the pilot flame and viscosity increasing caused by the pilot flame as well as the experimental uncertainty.

The velocity profiles of the coal flame A1 case show quite similar agreement and deviations as those of the pure pilot gas flame case, therefore, the detailed comparisons are attached in the supplementary materials for brevity. Figure 4 shows the comparisons of the velocities profiles from the LES results and the experiments for the coal flame A3 case. Under-predictions of the RMS velocities at the outer radial 


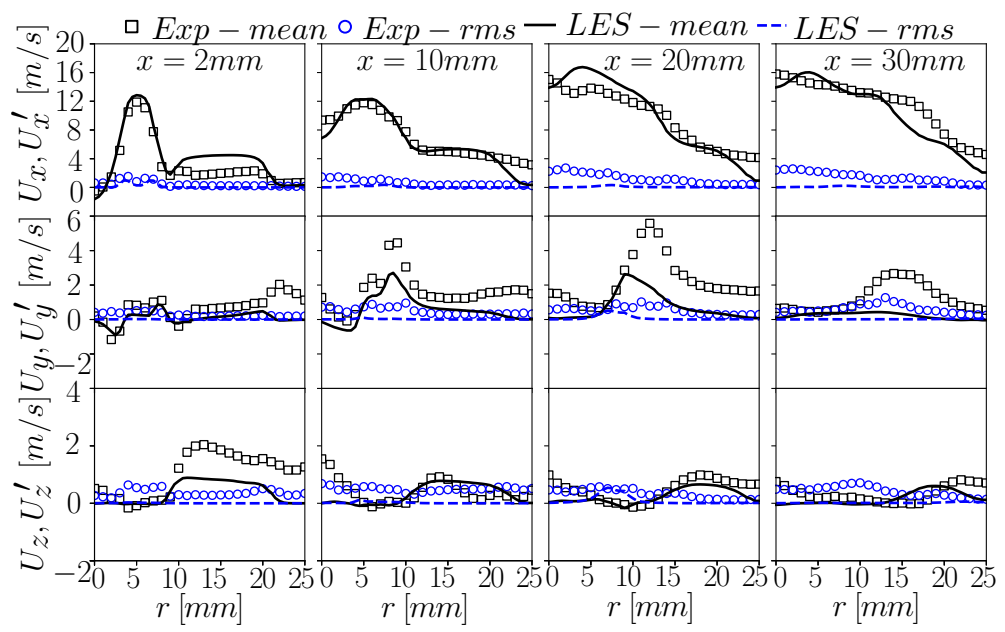

Figure 4: Comparisons of the mean and RMS velocities profiles of the LES results and experiments for the coal flame A3 case.

region $(10 \mathrm{~mm}<r<20 \mathrm{~mm})$ can still be found as the pure pilot gas flame, which is because there are very limited particles transported into the pilot flow in the near-nozzle region, and this deviation still remain even a turbulent pipe ring inlet is used for the pilot flow (see the supplementary materials). The mean axial velocity profiles are in a good agreement with the experimental at downstream locations 2, 10 and $20 \mathrm{~mm}$, while considerable under-prediction can be observed at $x=30 \mathrm{~mm}$ and $r>15 \mathrm{~mm} . U_{y}$ can generally reproduced at $\mathrm{x}=2$ and $10 \mathrm{~mm}$, while is significantly under-predicted at $\mathrm{x}=20$ and $30 \mathrm{~mm}$, which can be attribute to the boundary treatment of using a pipe ring turbulent flow to characterize the bluff-body effect and assuming pilot gas as a mixture of volatiles and carrier gas. By comparing the results of three reacting cases, it is interesting to find that the velocity profiles of the two coal flames predicted by the LES are quite similar with those of the pure pilot gas flame (especially at $x=2,10$, and $20 \mathrm{~mm}$ ). We further analyze the gas temperature field and particle devolatilization behavior to find possible reasons for this similarity. Figure 5 shows 


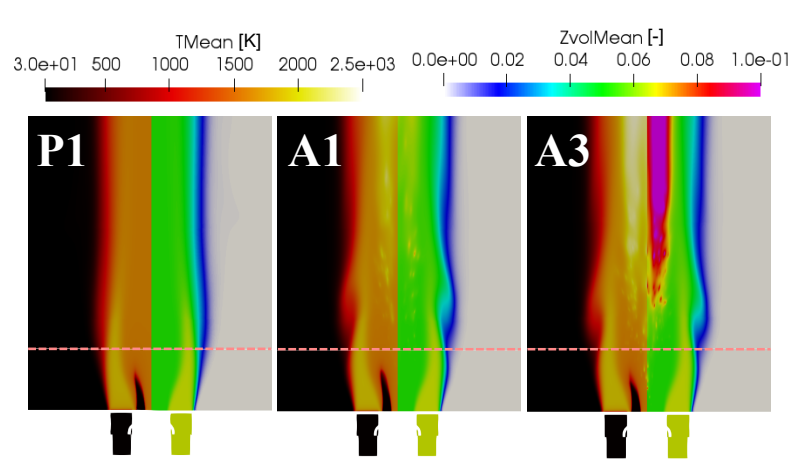

Figure 5: Contours of the mean gas temperature and mixture fraction of volatiles $\left(Z_{v o l}\right)$ at the near-nozzle region for pilot gas flame, coal flame A1 and A3. The red dash line represents the location $x=30 \mathrm{~mm}$.

the contours of the mean gas temperature and mixture fraction of volatiles $\left(Z_{v o l}\right)$ at the near-jet region for the pure pilot gas flame and two coal flames. It is found that those measurement locations $(x=2,10,20$ and $30 \mathrm{~mm})$ are too close to burner, and there are very limited volatiles released for the two coal flames in those locations. With limited volatiles released and burned, the gas temperature field does not change much compared with the pure pilot gas flame, resulting in a similar velocity profile. Form the LES results, some suggestions are made here for the future experimental measurement. The first one is that the particle and gas properties at more downstream locations should be measured. The previous experiments were conducted for the near-nozzle regions where coal combustion has limited effects on the flow field. The second one is that more combustion related scalar fields, such as major gas species, and gas and particle temperatures, should be measured for comprehensively evaluating the mode performance. 


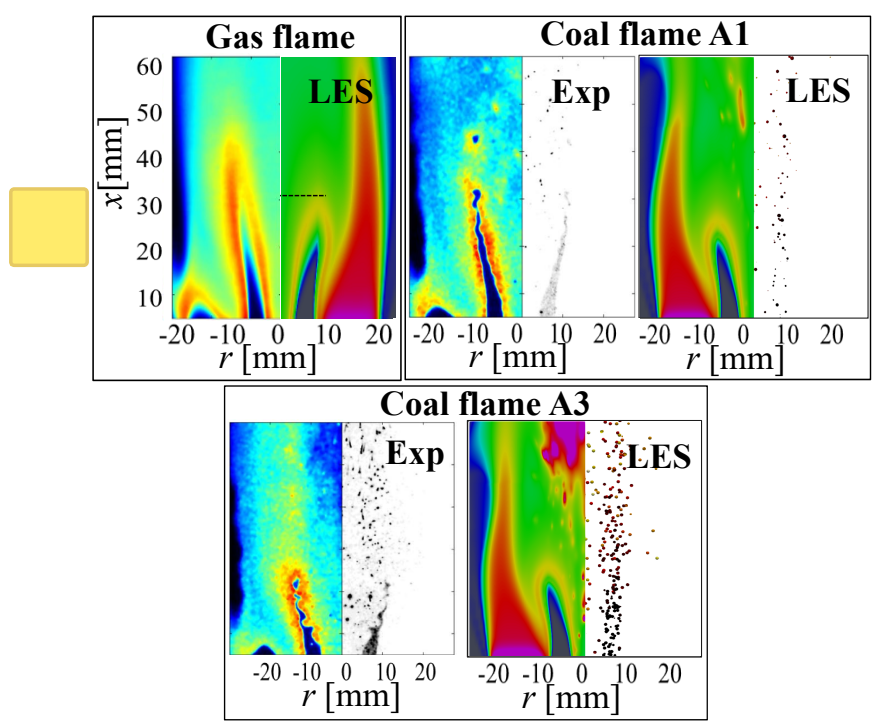

Figure 6: Comparisons of the instantaneous OH PLIF and Mie scatter images predicted with the LES and measured by experiment for the pure pilot gas flame, coal flame A1 and A3 cases.

\subsubsection{OH-PLIF and Mie scatter comparisons}

Comparisons of the instantaneous $\mathrm{OH}$ PLIF and Mie scatter images from the LES and the experiment for the pure pilot gas flame, coal flame A1 and A3 cases are displayed in Fig. 6. For pure pilot gas flame case, only the OH PLIF images are compared. It can be found that the peak value region for the pilot flame can be well reproduced, while the pilot flame length is over-predicted. The $\mathrm{OH}$ contour of the primary gas flame shows similar distribution as the experimental data with the same angle but under-predicted length. Those deviations can be attributed to the boundary treatment of assuming pilot methane/air gas as a mixture of volatiles and carrier gas in the model implementation, which would give quite different predictions of $\mathrm{OH}$ mass fraction, especially when heavy hydrocarbon is introduced into the volatile species $[31,32]$. This can be resolved by extending the present model to a three-mixture-fraction FPV model [33], which, however, is beyond the scope of the 
present paper. For the coal flame cases, the particle distribution can be reproduced as seen in the right columns of coal flame results in Fig. 6. A small part of particles are transported into the recirculation zone induced by the bluff-body. While coal particles do not move much to high radius regions due to the under-predicted turbulence. Compared with the pure pilot gas flame, similar $\mathrm{OH}$ distributions of the pilot flames can be observed for both the coal flames since coal particles have not been transported into the pilot radial region in the near-nozzle region. The $\mathrm{OH}$ contours of the primary coal flames share similar expansion angles as those of the experiments with under-predicted height in the coal flame A1 case and well matched height in the coal flame A3 case. It is also found that at the downstream regions $(\mathrm{x}>50 \mathrm{~mm})$ of the LES results, there are some $\mathrm{OH}$ produced by the ignited volatiles, and this phenomenon is more pronounced at coal flame A3 case due to the higher coal load and more released volatiles.

\subsubsection{Coal particle properties}

Figure 7 shows the instantaneous particle scatters of the remaining char and volatile mass fractions as a function of the residence time. It is found that small particles devolatilize earlier and faster, and the devolatilization process is completed at residence time of $5 \mathrm{~ms}$, while large particles devolatilize more slowly with the devolatilization process completed at $15 \mathrm{~ms}$. The mean char fraction first increases when the devolatilization process starts, and begins to decrease when the small particles are fully devolatilized and char in small particles begins to be oxidized. Char in small particles have been fully oxidized at a residence time of $20 \mathrm{~ms}$, while for large particles, much smaller char burnout can be found even at a residence time of $50 \mathrm{~ms}$. Figure 8 compares the ensemble mean volatiles and char fractions of particles in a basis of the their initial total mass. It is found that the increasing of coal load has 

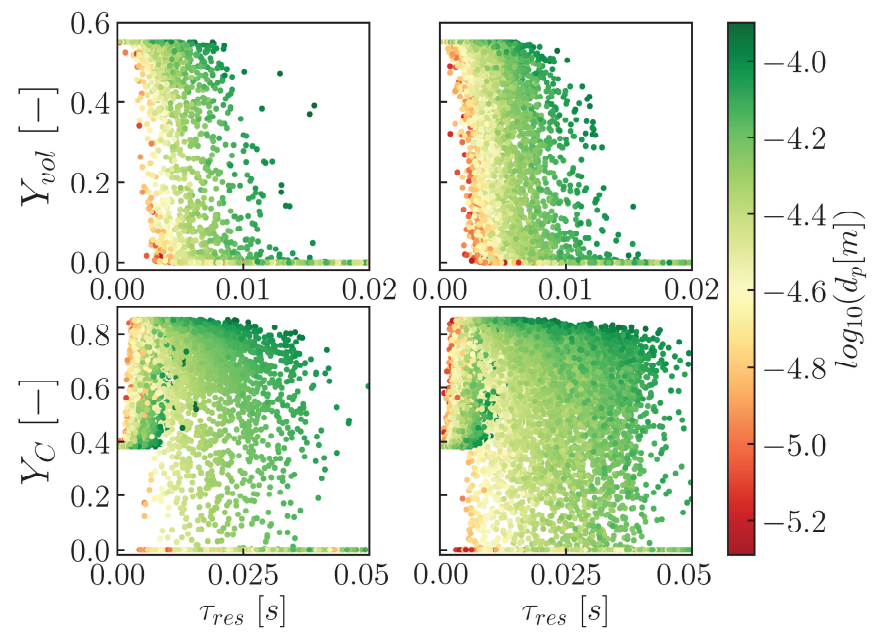

Figure 7: Instantaneous particle scatters of the char and volatile mass fractions as a function of the residence time. Particles are colored with the log-scale exponent of their diameters. The left and right columns donate the coal flame A1 and A3 results, respectively.

limited effect on the particle devolatilization process, but shows slight reduction for the char combustion process. This is because that with the increasing of coal load, there are more volatiles released and oxidized with less oxygen remained, resulting in an lower char oxidization rate.

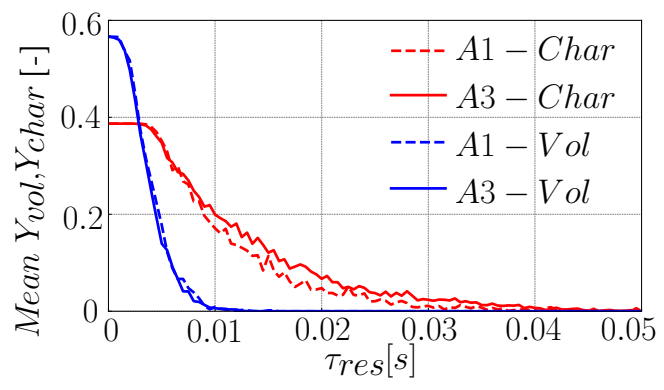

Figure 8: Mean volatile and char mass fractions in a basis of the initial particle mass. 


\section{Conclusions}

In the present study, we report the first LES study of the Cambridge CCB2 coal flames, one of the target flames in CBC workshop, with an extended two-mixturefraction FPV model. The results show the present LES/FPV can generally reproduce the flow field and particle distribution (Mie scatter images), while there are considerable deviations in the $\mathrm{OH}$ comparisons due to using a mixture of volatiles and carrier gas to replace the methane-air/methane-carrier-gas mixtures in the primary/pilot flow. This indicates for such gas-assisted coal flames, the pilot fuel stream needs to be rigorously considered in the flamelet tabulation, which can be resolved by extending the two-mixture-fraction model into a three-mixture-fraction model that will be studied in the future. The instantaneous Lagrangian particles histories show that the increasing of coal load has a negligible effect on devolatilization, but delays the char conversion. Finally, more combustion-related scalars are expected to be measured for comprehensively evaluating the performance of the extended FPV model in the future work.

\section{Acknowledgments}

The authors are grateful for the support from the National Natural and Science Foundation (Grant No: 91741203) and National Key Research and Development Program of China (Grant: 2017YFB0601805).

\section{References}

[1] A. Williams, M. Pourkashanian, J. Jones, Combustion of pulverised coal and biomass, Progr. Energ Combust. Sci., 27 (2001) 587-610. 
[2] O.T. Stein, G. Olenik, A. Kronenburg, et al., Towards comprehensive coal combustion modelling for LES. Flow, Turbul. Combust. 90 (2013) 859-84.

[3] K. Luo, H.O. Wang, J.R. Fan, F.X. Yi, Direct Numerical Simulation of Pulverized Coal Combustion in a Hot Vitiated Co-flow, Energy fuels 26 (2012) 6128-6136.

[4] Coal and Biomass Conversion (CBC) workshop, 2019, available at http: //www . cbc.uni-due.de/?file=workshop.

[5] S. Hwang, R. Kurose, F. Akamatsu, et al., Application of optical diagnostics techniques to laboratory-scale turbulent pulverized coal flame. Energy fuels 19 (2005) 38292.

[6] S. Balusamy, A. Schmidt, S. Hochgreb, Flow field measurements of pulverized coal combustion using optical diagnostic techniques, Exp Fluids 54 (2013) 1534.

[7] S. Balusamy, M. Kamal, S.M. Lowe, et al., Laser diagnostics ofpulverized coal combustion in $\mathrm{O}_{2} / 2$ and $\mathrm{O}_{2} / \mathrm{CO}_{2}$ conditions: velocity and scalar field measurements, Exp Fluids 56 (2015) 108.

[8] X. Wen, K. Luo, H.H. Jin, J.R. Fan, Large eddy simulation of piloted pulverised coal combustion using extended flamelet/progress variable model, Combust. Theor. Model. 21 (2017) 925-953.

[9] B.M. Franchetti, F.C. Marincola, S. Martinez, A.M. Kempf, Large Eddy simulation of a pulverised coal jet flame, Proc. Combust. Inst. 34 (2013) 2419-2426.

[10] M. Rieth, A.G. Clements, M. Rabacal, et al., Flamelet LES modeling of coal combustion with detailed devolatilization by directly coupled CPD, Proc. Combust. Inst. 36 (2017) 2181-2189. 
[11] A. Farazi, A. Attili, A. Kang, Numerical study of coal particle ignition in air and oxy-atmosphere, Proc. Combust. Inst. 37 (2019) 2867-2874.

[12] K.D. Wan, J. Xia, Z.H. Wang, et al., Online-CPD-Coupled Large-Eddy Simulation of Pulverized-Coal Pyrolysis in a Hot Turbulent Nitrogen Jet, Combust. Sci. Technol. 189 (2017) 103-131.

[13] J. Watanabe, K. Yamamoto, Flamelet model for pulverized coal combustion, Pro. Combust. Inst. 35 (2015) 2315-2322.

[14] X.Y. Zhao, D.C. Haworth, Transported PDF modeling of pulverized coal jet flames, Combust. Flame 161 (2014) 1866-1882.

[15] L.F. Zhao, M.J. Cleary, O.T. Stein, A. Kronenburg, A two-phase MMC-LES model for pyrolysing solid particles in a turbulent flame, Combust. Flame 209 (2019) 322-336.

[16] S.P. Domino, P.J. Smith, State space sensitivity to a prescribed probability density function shape in coal combustion systems: Joint -PDF versus clipped Gaussian PDF, Pro. Combust. Inst. 28 (2000) 2329-2326.

[17] M. Muto, H. Watanabe, R. Kurose, et al., Large-eddy simulation of pulverized coal jet flame - Effect of oxygen concentration on NOx formation, Fuel 142 (2015) 152-163.

[18] X. Wen, H.O Wang, K. Luo, J.F. Fan, Evaluation of flamelet/progress variable model for laminar pulverized coal combustion, Phys. Fluids 29 (2017) 083607.

[19] S. Badzioch, P.G. Hawksley, Kinetics of thermal decomposition of pulverized coal particles, Indus. Eng. Chem. Process Des. Develop., 9 (1970) 521-530. 
[20] T.H. Fletcher, A.R. Kerstein, R.J. Pugmire, et al., Chemical percolation model for devolatilization. 3. Direct use of C-13 NMR data to predict effects of coal type, Energy fuels 6 (1992) 414-431.

[21] M. Reith, A.M. Kempf, A. Kronenburg, O.T. Stein, Carrier-phase DNS of pulverized coal particle ignition and volatile burning in a turbulent mixing layer, Fuel 212 (2018) 364-374.

[22] M. Baum, P. Street, Predicting the combustion behaviour of coal particles, Combust. Sci. Technol. 3 (5) (1971) 231-243.

[23] J. Smagorinsky, General circulation experiments with the primitive equations: I. the basic experiment, Mon. Weather Rev., 91 (3) (1963) 99-164.

[24] X. Wen, K. Luo, H.O. Wang, J.R. Fan, Analysis of pulverized coal flame stabilized in a 3D laminar counterflow, Combust. Flame 189 (2018) 106-125.

[25] P. Cheng, Two-dimensional radiating gas flow by a moment method, AIAA J. 2 (9) (1964) 1662-1664.

[26] H. Pitsch, Flamemaster: A C++ computer program for 0D combustion and 1D laminar flame calculations, (1998).

[27] G.L. Tufano, O.T. Stein, A. Kroonenburg, et al., Resolved flow simulation of pulverized coal particle devolatilization and ignition in air- and O2/CO2atmospheres, Fuel 186 (2016) 285-292.

[28] Cambridge coal flame database. Available at: http://www-g.eng.cam.ac.uk/ reactingflows/databases/coal/. (Lase accessed at 7.10.2019) 
[29] X. Wen, K. Luo, Y.J. Luo, H.O. Wang, J.R. Fan, Large-eddy simulation of multiphase combustion jet in cross-flow using flamelet model, Int. J. Multiphas. Flow 108 (2018) 211-225.

[30] J.K. Xing, K. Luo, P. Heinz, et al., Predicting kinetic parameters for coal devolatilization by means of Artificial Neural Networks, Proc. Combust. Inst. 37 (2019) 2943-2950.

[31] M. Xia, D. Zabrodiec, P. Scouflaire, B. Fiorina, N. Darabiha, Experimental and numerical studies of pulverized coal devolatilization and oxidation in strained methane/air flames, Proc. Combust. Inst. 36 (2017) 2123-2130.

[32] J.K. Xing, K. Luo, H.O. Wang, et al., Comparative study on different treatments of coal devolatilization for coal combustion simulation, Energy fuels 34 (2020) 3816-3827.

[33] X. Wen, Y.J. Luo, H.O. Wang, et al., A three mixture fraction flamelet model for multi-stream laminar pulverized coal combustion, Pro. Combust. Inst. 37 (2019) 2901-2910. 